\title{
ON PRINCIPAL SOLUTIONS OF LINEAR DIFFERENTIAL EQUATIONS
}

\author{
STEVEN BANK
}

1. Introduction. In this paper we investigate the asymptotic behavior of solutions of certain $n$th order nonhomogeneous linear ordinary differential equations, $\Omega(y)=\phi$, near a singular point at $\infty$. The class of $n$th order linear differential operators, $\Omega$, treated here consists roughly of those whose coefficients are complex functions, defined and analytic in unbounded sectorial regions, and have asymptotic expansions as $x \rightarrow \infty$ in terms of real (but not necessarily integral) powers of $x$ and/or functions (called trivial) which are of smaller rate of growth ( $\prec$ ) than all powers of $x$ as $x \rightarrow \infty$. (We are using here the concept of asymptotic equivalence $(\sim)$ as $x \rightarrow \infty$ and the order relations " $\prec$ " introduced in $[3, \S 13]$. However, it should be noted (see $[3, \S 128(\mathrm{~g})]$ ) that the class of operators treated here includes as a special case those operators where no requirement is imposed except that each coefficient be analytic and have an asymptotic expansion (in the customary sense) of the form $\sum C_{j} x^{-\lambda_{j}}$ with $\lambda_{j}$ real and $\lambda_{j} \rightarrow+\infty$ as $j \rightarrow \infty$. (A summary of the necessary definitions from [3] appears in $\$ 2$ below.)

In [5], Strodt showed that if $\phi$ is a nontrivial analytic function which also possesses, as $x \rightarrow \infty$, an asymptotic expansion in terms of real powers of $x$ and/or trivial functions, then the equation $\Omega(y)=\phi$ has at least one solution $y_{0}$ which is $\sim$ to a logarithmic monomial (i.e., a function of the form $K x^{\alpha_{0}}(\log x)^{\alpha_{1}}(\log \log x)^{\alpha_{2}} \cdots\left(\log _{q} x\right)^{\alpha_{q}}$ for complex $K \neq 0$ and real $\alpha_{j}$ ) and such that if $f \prec y_{0}$, then $\Omega(f) \prec \phi$. (A solution with these two properties is called a principal solution in $[3, \S 69]$ and is clearly of minimal rate of growth at $\infty$.)

In this paper we consider the case where $\phi$ is any function $\sim$ to a logarithmic monomial, and we show in $\S 3$ that the equation $\Omega(y)=\phi$ always has a principal solution. As a corollary (\$5) we apply a result proved in $[1, \S 12]$ to obtain a representation theorem for those solutions of $\Omega(y)=\phi$ which are $\prec$ some power of $x$. Our method of proving $\$ 3$ consists of first obtaining a sufficiently close approximate solution by successive integrations of a factored equation, and then using the approximate solution to transform the equation in to one in which an exact solution can be obtained using $[4, \S 99]$.

2. Concepts from [3]. (a) $[3, \S 94]$. Let $-\pi \leqq a \leqq b \leqq \pi$. For each non-

Received by the editors March 2, 1967. 
negative real-valued function $g$ on $(0,(b-a) / 2)$, let $V(g)$ be the union (over $\delta \in(0,(b-a) / 2)$ ) of all sectors $a+\delta<\arg (x-h(\delta))<b-\delta$ where $h(\delta)=g(\delta) \exp (i(a+b) / 2)$. The set of all $V(g)$ (for all choices of $g$ ) is denoted $F(a, b)$ and is a filter base which converges to $\infty$. A statement is said to hold except in finitely many directions (briefly, e.f.d.) in $F(a, b)$ if there are finitely many points $r_{1}<\cdots<r_{q}$ in $(a, b)$ such that the statement holds in each of $F\left(a, r_{1}\right), F\left(r_{1}, r_{2}\right)$, ..., $F\left(r_{q}, b\right)$ separately.

(b) $[3, \S 13]$. If $f$ is analytic in some $V(g)$, then $f \rightarrow 0$ in $F(a, b)$ means that for any $\epsilon>0$, there is a $g_{1}$ such that $|f(x)|<\epsilon$ for all $x \in V\left(g_{1}\right)$. $f \prec 1$ means that in addition to $f \rightarrow 0$, all functions $\theta_{j}^{k} f \rightarrow 0$ where $\theta_{j} f$ $=x \log x \cdots \log _{j-1} x f^{\prime}$ and where $\theta_{j}^{k}$ is the $k$ th iteration of the operator $\theta_{j}$. Then $f_{1} \prec f_{2}, f_{1} \sim f_{2}, f_{1} \approx f_{2}$ mean respectively $f_{1} / f_{2} \prec 1, f_{1}$ $-f_{2} \prec f_{2}$ and $f_{1} \sim c f_{2}$ for some constant $c \neq 0$. If $f \sim c$, we write $f(\infty)=c$, while if $f \prec 1$, we write $f(\infty)=0$. If $M=x^{\alpha_{0}}(\log x)^{\alpha_{1}} \ldots\left(\log _{r} x\right)^{\alpha_{r}}$ for some $r$ and $M$ is not constant, then by $[3, \S 28] f \prec M$ implies $f^{\prime} \prec M^{\prime}$. If $f \approx M$, then $\delta_{k}(f)$ will denote $\alpha_{k}$. If $j \geqq i$, then $s_{j i}(\alpha)$ will denote the elementary symmetric function of degree $i$ in $\alpha, \alpha-1, \cdots$, $\alpha-j+1$.

(c) $[3, \S 49]$. A logarithmic domain of rank zero (briefly an $L D_{0}$ ) over $F(a, b)$ is a complex vector space $E$ of functions (each analytic in some $V(g)$ ) which contains the constants and such that any finite linear combination of elements of $E$, with coefficients which for some $q \geqq 0$ are functions of the form $c x^{\alpha_{0}}(\log x)^{\alpha_{1}} \cdots\left(\log _{q} x\right)^{\alpha_{q}}$ (for real $\alpha_{j}$ ), is either $\sim$ to a function of this latter form or is trivial.

3. The main theorem. Consider the equation $\Omega(y)=\phi$, where $\Omega(y)$ is an $n$th order linear differential polynomial with coefficients in an $L D_{0}$ over $F(a, b)$, and where $\phi$ is a function which in $F(a, b)$ is $\sim$ to a logarithmic monomial. If $\theta$ is the operator $\theta y=x y^{\prime}, \Omega(y)$ may be written $\Omega(y)=\sum_{i=0}^{n} B_{i}(x) \theta^{i} y$, where the functions $B_{j}$ belong to an $L D_{0}$. We assume $B_{n}$ is nontrivial. By dividing the equation $\Omega(y)=\phi$ through by the highest power of $x$ which is $\sim$ to a coefficient $B_{j}$, we may assume that for some $m \geqq 0, B_{m} \approx 1$ while for each $j, B_{j} \prec 1$ or $B_{j} \approx 1$. Let $F(\alpha)$ $=\sum_{i=0}^{n} B_{i}(\infty) \alpha^{i}$. Let $Q$ be the logarithmic monomial such that $\phi \sim Q$ and let $\delta_{j}(Q)=\sigma_{j}$ for each $j$. Define a logarithmic monomial $M$ as follows. If $F\left(\sigma_{0}\right) \neq 0$, let $M=\left(F\left(\sigma_{0}\right)\right)^{-1} Q$. If $\sigma_{0}$ is a root of $F$ of multiplicity $r$, then let

$$
\begin{aligned}
M=\left(F^{(r)}\left(\sigma_{0}\right) / r !\right)^{-1}\left(s_{r r}\left(\sigma_{1}+r\right)\right)^{-1}(\log x)^{r} Q \\
\text { if } \sigma_{1} \notin\{-1,-2, \cdots,-r\},
\end{aligned}
$$

while if $\sigma_{1} \in\{-1,-2, \cdots,-r\}$, let 


$$
M=c(\log x)^{r}\left(\log _{2} x \cdots \log _{k} x\right) Q
$$

where $k=\min \left\{j: j \geqq 2, \sigma_{j} \neq-1\right\}$, and

$$
c=\left(s_{r, r-1}\left(\sigma_{1}+r\right)\right)^{-1}\left(\sigma_{k}+1\right)^{-1}\left(F^{(r)}\left(\sigma_{0}\right) / r !\right)^{-1} .
$$

Then: (1) The equation $\Omega(y)=\phi$ possesses at least one solution $y_{0}$ $\sim M$ e.f.d. in $F(a, b)$. (2) If $y_{0}$ is a solution of $\Omega(y)=\phi$ such that $y_{0} \sim M$ in some $F\left(a_{1}, b_{1}\right)$, then for any function $f$ which is $\prec y_{0}$ in $F\left(a_{1}, b_{1}\right)$, we have $\Omega(f) \prec \phi$ in $F\left(a_{1}, b_{1}\right)$. In particular, among all solutions of $\Omega(y)=\phi$ in $F\left(a_{1}, b_{1}\right), y_{0}$ is of minimal rate of growth at $\infty$.

PROOF OF PART (2). We consider $\Omega(y)-Q$ and apply the algorithm introduced in $[3, \S 66]$ which produces the set of those logarithmic monomials $N$ (called principal monomials in [3, \$67]) such that $\Omega(N) \sim Q$ and $\Omega(f) \prec Q$ whenever $f \prec N$. For $\Omega(y)-Q$ we find by applying the algorithm that $M$ is the unique principal monomial. Hence if $f \prec M$, then $\Omega(f) \prec Q$. Since $y_{0} \sim M$ and $\phi \sim Q$, part (2) clearly follows.

The proof of part (1) will be based on a sequence of lemmas and will be concluded in $\S 6$.

4. Lemma. Let $\gamma$ be a complex number and let $\psi$ be a function which in $F(a, b)$ is $\sim$ to a logarithmic monomial $R$. Let $\delta_{i}(R)=\lambda_{i}$. Define a logarithmic monomial $N$ as follows:

(a) If $\lambda_{0} \neq \gamma$, let $N=\left(\lambda_{0}-\gamma\right)^{-1} R$.

(b) If $\lambda_{0}=\gamma$, let $N=\left(\lambda_{q}+1\right)^{-1}\left(\log x \cdots \log _{q} x\right) R$ where $q=$ $\min \left\{j: j \geqq 1, \lambda_{j} \neq-1\right\}$.

Then in $F(a, b)$, the equation $x y^{\prime}-\gamma y=\psi$ has at least one solution $y^{*} \sim N$.

Proof. Under the change of variable $y=x^{\lambda} 0 z$ and multiplication by $x^{-\lambda_{0}}$, the equation $x y^{\prime}-\gamma y=\psi$ becomes

$$
x z^{\prime}+\left(\lambda_{0}-\gamma\right) z=\psi_{0}
$$

where $\psi_{0}=x^{-\lambda} 0 \psi$.

Let $N_{0}=x^{-\lambda_{0}} N$. The proof is divided into three cases.

Case A. $\operatorname{Re}\left(\lambda_{0}-\gamma\right) \neq 0$.

In this case, under $z=N_{0}+N_{0} w$ and division by $\left(\lambda_{0}-\gamma\right) N_{0}$, equation (1) becomes,

$$
x\left(\lambda_{0}-\gamma\right)^{-1} w^{\prime}+f(x) w=g(x),
$$

where $f \sim 1$ (since $x N_{0}^{\prime} \prec N_{0}$ by a simple calculation) and where $g \prec 1$ since $\left(\lambda_{0}-\gamma\right) N \sim \psi$. Thus (2) is normal in the sense of $[3, \S 83]$ with divergence monomial $\left(\lambda_{0}-\gamma\right) x^{-1}$. Since $d=\operatorname{Re}\left(\lambda_{0}-\gamma\right) \neq 0$, it follows 
from $[3, \S 111]$ (when $d>0$ ) and $[3, \S 117]$ (when $d<0$ ) that $(2)$ possesses a solution $w_{0} \prec 1$ in $F(a, b)$. Then clearly $y^{*}=x^{\lambda_{0}}\left(N_{0}+N_{0} w_{0}\right)$ is $\sim N$ and satisfies the equation $x y^{\prime}-\gamma y=\psi$.

Case B. $\lambda_{0}=\gamma$.

Thus (1) is of the form $z^{\prime}=x^{-1} \psi_{0}$. With $N$ as defined in (b) above, it is proved in $\left[2\right.$, p. 272] that for some constant $A, z_{0}=A+\int_{x_{0}}^{x} x^{-1} \psi_{0}$ is $\sim N_{0}$ in $F(a, b)$. Hence if $y^{*}=x^{\lambda_{0}} z_{0}$, then $y^{*}$ satisfies the conclusion.

Case C. $\operatorname{Re}\left(\lambda_{0}-\gamma\right)=0$ and $\lambda_{0} \neq \gamma$.

In this case, (1) may be written $x z^{\prime}-\sigma i z=\psi_{0}$ where $\sigma=i\left(\lambda_{0}-\gamma\right)$ is a nonzero real number. Under $z=-(\sigma i)^{-1} \psi_{0}+w$, this becomes $x w^{\prime}-\sigma i w=\psi_{1} \quad$ where $\psi_{1}=(\sigma i)^{-1} x \psi_{0}^{\prime}$. Since $\psi_{0} \prec(\log x)^{\lambda_{1}+1 / 2}, \quad \psi_{1}$ $\prec(\log x)^{\lambda_{1}-1 / 2}$ by $\S 2(\mathrm{~b})$, and hence $\psi_{1} \prec \psi_{0}$ since $(\log x)^{\lambda_{1}-\epsilon} \prec \psi_{0}$ for all $\epsilon>0$. Under $w=-(\sigma i)^{-1} \psi_{1}+u$, we obtain $x u^{\prime}-\sigma i u=\psi_{2}$ where by $\S 2(\mathrm{~b}), \psi_{2} \prec(\log x)^{\lambda_{1}-3 / 2}$ (thus $\psi_{2} \prec \psi_{0}$ since $\psi_{0} \succ(\log x)^{\lambda_{1}-\epsilon}$ for all $\epsilon>0$ ). Clearly this process can be repeated so as to make the constant term $\prec(\log x)^{\alpha}$ for $\alpha$ as small as desired. Hence there is a function $f \sim-(\sigma i)^{-1} \psi_{0}$ in $F(a, b)$ such that under $z=f+v$, equation (1) becomes

$$
x v^{\prime}-\sigma i v=\phi_{1}
$$

where $\phi_{1}$ is chosen so that,

$$
\phi_{1} \prec(\log x)^{-1-t}
$$

where $t=1+\max \left\{0,-2 \lambda_{1}\right\}$.

The technique we now employ to prove the existence of a solution $v_{0} \prec f$ of (3) is similar to the technique used by Strodt in the proof of [6, Section 107].

Let $E_{1} \in F(a, b)$ be such that on $E_{1}$, we have $|x| \geqq 2$ and

$$
\left|\phi_{1}(x)\right| \leqq(\log |x|)^{-1-t / 2} \text {. }
$$

For $x$ and $x_{1}$ in $E_{1}$, let $B\left(x, x_{1}\right)=\exp \int_{x}^{x_{1}}(-\sigma i / u) d u$, where the contour is any rectifiable path from $x$ to $x_{1}$ in $E_{1}$. Then clearly, if we put $L(x, \rho)=B(x, \rho x)$ for $1 \leqq \rho<\infty$, we have

$$
|L(x, \rho)| \equiv 1 \text { and } \partial L(x, \rho) / \partial x \equiv 0 .
$$

Hence,

$$
|L(x, \rho)| \rho^{-1}\left|\phi_{1}(\rho x)\right| \leqq(\rho \log 2 \rho)^{-1}(\log 2 \rho)^{-t / 2}
$$

for $x \in E_{1}$ and $1 \leqq \rho<\infty$; and since the right side is

$$
(-2 / t) d\left((\log 2 \rho)^{-t / 2}\right) / d \rho,
$$

we have by the $M$-test $[7$, p. 22] that the integral 


$$
v_{0}(x)=-\int_{1}^{\infty} L(x, \rho) \rho^{-1} \phi_{1}(\rho x) d \rho
$$

is uniformly convergent on $E_{1}$ and thus represents an analytic function there (e.g., [7, p. 100]) whose derivative may be calculated by differentiating under the integral sign. In view of (5), clearly

$$
\left|v_{0}(x)\right| \leqq(2 / t)(\log |x|)^{-t / 2}
$$

on $E_{1}$ and hence $v_{0} \rightarrow 0$ in $F(a, b)$. Differentiating (7), we see easily that $v_{0}^{\prime}-\sigma i x^{-1} v_{0}=x^{-1} \phi_{1}$ in $E_{1}$, so $v_{0}$ is a solution of (3). Successively differentiating (7) and using (6), we see that for all $j$

$$
\theta^{j_{v_{0}}}(x)=-\int_{1}^{\infty} L(x, \rho) \rho^{-1}\left(\theta^{j} \phi_{1}\right)(x \rho) d \rho
$$

(where, for example, $\left(\theta \phi_{1}\right)(x \rho)=x \rho \phi_{1}^{\prime}(x \rho)$ etc.) in $E_{1}$. Since $\phi_{1}$ $\prec(\log x)^{-1-t / 2}$, it follows (see $\left.\S 2(\mathrm{~b})\right)$ that $\theta^{j} \phi_{1} \prec(\log x)^{-(j+1)-t / 2}$ in $F(a, b)$, and so, for each $j$, there is an $S_{j} \in F(a, b)$ and a constant $c_{j}$ such that

$$
\left|\theta^{j} \phi_{1}(x)\right| \leqq c_{j}(\log |x|)^{-(j+1)-t / 2} \text { on } S_{j} .
$$

Hence by (8a), there is a $C_{j}^{\prime}$ such that

$$
\left|\theta^{j_{v_{0}}}(x)\right| \leqq C_{j}^{\prime}(\log |x|)^{-j-t / 2} \text { in } S_{j} .
$$

Thus $\theta^{j} v_{0} \rightarrow 0$ in $F(a, b)$ for each $j$. Now let $p>1$. Then, by the definition of the operator $\theta_{p}, \theta_{p} v_{0}=G \theta v_{0}$ where $G=\log x \cdots \log _{p-1} x$. It is routine to verify by induction on $j$ that for $j=1,2, \ldots$

$$
\theta_{p}^{j} v_{0}=\sum_{\alpha=1}^{j} G_{\alpha j} \theta^{\alpha} v_{0}
$$

where

$$
G_{\alpha j}=\sum m\left(i_{1}, \cdots, i_{j}, \alpha\right) G^{i_{1}}(\theta G)^{i_{2}} \cdots\left(\theta^{j-1} G\right)^{i}
$$

in which the $m$ 's are constants, $i_{1}+\cdots+i_{j}=j$ and $i_{2}+2 i_{3}+\cdots$ $+(j-1) i_{j}=j-\alpha$ for each term in (11). Now for all $\epsilon>0, G \prec(\log x)^{1+\epsilon}$ so (see $\S 2(\mathrm{~b})) \theta^{j} G \prec(\log x)^{1-j+\epsilon}$ for each $j$. Hence, by (11), for each $\alpha$ and $j$ we have $G_{\alpha j} \prec(\log x)^{\alpha+\epsilon j}$ for all $\epsilon>0$. Now $t$ is a fixed positive number and so for each given $\alpha$ and $j$, we have, by taking $\epsilon=t / 5 j$, that $G_{\alpha j} \prec(\log x)^{\alpha+t / 4}$. Hence there exist $S_{\alpha j} \in F(a, b)$ and constants $d(\alpha, j)$ such that on $S_{\alpha j}$,

$$
\left|G_{\alpha j}(x)\right| \leqq d(\alpha, j)(\log |x|)^{\alpha+t / 4} .
$$

Thus by (9) and (10), for each $p$ and $j$, 


$$
\left|\theta_{p}^{j} v_{0}(x)\right| \leqq m_{p j}(\log |x|)^{-t / 4}
$$

in some element of $F(a, b)$ for some constant $m_{p j}$. Thus $\theta_{p}^{j} v_{0} \rightarrow 0$ for all $p$ and $j$ since $t>0$ and so

$$
v_{0} \prec 1 \text { in } F(a, b) .
$$

Since $v_{0}$ solves (3), we have

$$
v_{0}=(\sigma i)^{-1}\left(x v_{0}^{\prime}-\phi_{1}\right) .
$$

Since $v_{0} \prec 1, x v_{0}^{\prime} \prec(\log x)^{-1}$. Thus since $\phi_{1} \prec(\log x)^{-1-t / 2}$, we have by (13) that $v_{0} \prec(\log x)^{-1}$. Hence $x v_{0}^{\prime} \prec(\log x)^{-2}$, and so if $-1-t / 2$ $<-2$, we have $v_{0} \prec(\log x)^{-2}$. Continuing this way, if $m$ is the greatest integer $<1+t / 2$, then $v_{0} \prec(\log x)^{-m}$, and so since $m+1 \geqq 1+t / 2$, we have by (13) that $v_{0} \prec(\log x)^{-1-t / 2}$ in $F(a, b)$. Thus by (4), $v_{0}$ $\prec(\log x)^{\lambda_{1}-1}$ and so $v_{0} \prec f$ in $F(a, b)$. Hence if $z_{0}=f+v_{0}$, then $z_{0} \sim f$ and $z_{0}$ solves (1). Hence $y^{*}=x^{\lambda} z_{0}$ is a solution of $x y^{\prime}-\gamma y=\psi$ and $y^{*} \sim N$ in $F(a, b)$ concluding the proof.

5. Lemma. Assume the hypothesis and notation of \$3. Let $\Phi(y)$ $=\sum_{i=0}^{n} B_{i}(\infty) \theta^{i} y$. Then there exists a function $y^{*}$ such that $\Phi\left(y^{*}\right)=\phi$ and $y^{*} \sim M$ in $F(a, b)$ (where $M$ is as in $\S 3$ ).

Proof. Let $F(\alpha)=\sum B_{i}(\infty) \alpha^{i}$ be of degree $p$. If $p=0$, take $y^{*}$ $=\left(B_{0}(\infty)\right)^{-1} \phi$. Hence we may assume $p>0$. It is easy to verify that if $F(\alpha)=b_{p}\left(\alpha-\alpha_{1}\right) \cdots\left(\alpha-\alpha_{p}\right) \quad\left(\right.$ where $\left.b_{p}=B_{p}(\infty)\right)$, then $b_{p}^{-1} \Phi$ $=\left(\theta-\alpha_{1}\right) \circ \cdots \circ\left(\theta-\alpha_{p}\right)$ where the order of the factors is immaterial. Let $\phi^{*}=b_{p}^{-1} \phi$ and let $Q^{*}=b_{p}^{-1} Q$. We solve $\Phi(y)=\phi$ by successive integrations on $b_{p}^{-1} \Phi=\phi^{*}$ using $\S 4$, and we adopt the following notation. We let $y_{1}$ be any solution of $x y^{\prime}-\alpha_{1} y=\phi^{*}$ given by $\S 4$. Since $y_{1}$ is $\sim$ to a logarithmic monomial, we let $y_{2}$ be any solution of $x y^{\prime}-\alpha_{2} y=y_{1}$ given by $\S 4$. In general, $y_{j+1}$ is any solution of $x y^{\prime}$ $-\alpha_{j+1} y=y_{j}(1 \leqq j \leqq p-1)$ given by $\S 4$. Then clearly $y^{*}=y_{p}$ solves $\Phi(y)=\phi$. We will show $y^{*} \sim M$.

Case I. $F\left(\sigma_{0}\right) \neq 0$. By $\$ 4(\mathrm{a}), \quad y_{1} \sim\left(\sigma_{0}-\alpha_{1}\right)^{-1} Q^{*}$. Similarly $y_{2}$ $\sim\left(\sigma_{0}-\alpha_{2}\right)^{-1}\left(\sigma_{0}-\alpha_{1}\right)^{-1} Q^{*}$. Continuing by $\S 4(\mathrm{a}), y_{p} \sim\left(F\left(\sigma_{0}\right)\right)^{-1} Q$ so $y_{p} \sim M$.

Case II. $\sigma_{0}$ is a root of $F$ of multiplicity $r$ and $\sigma_{1} \notin\{-1, \cdots,-r\}$. Let $\alpha_{1}=\cdots=\alpha_{r}=\sigma_{0}$. By $\S 4(\mathrm{~b}), y_{1} \sim\left(\sigma_{1}+1\right)^{-1}(\log x) Q^{*}$. Similarly by $\$ 4(b)$,

$$
y_{j} \sim\left(\sigma_{1}+j\right)^{-1} \cdots\left(\sigma_{1}+1\right)^{-1}(\log x)^{j} Q^{*} \quad \text { for } 2 \leqq j \leqq r .
$$

Then by $\$ 4(\mathrm{a})$,

$$
y_{r+1} \sim\left(\sigma_{0}-\alpha_{r+1}\right)^{-1}\left(\sigma_{1}+r\right)^{-1} \cdots\left(\sigma_{1}+1\right)^{-1}(\log x)^{r} Q^{*}
$$


and by continuing to use $\S 4(\mathrm{a})$, clearly

$$
y_{p} \sim K(\log x)^{r} Q \quad \text { where } K=\left(F^{(r)}\left(\sigma_{0}\right) / r !\right)^{-1}\left(s_{r r}\left(\sigma_{1}+r\right)\right)^{-1}
$$

so $y_{p} \sim M$.

Case III. $\sigma_{0}$ is a root of $F$ of multiplicity $r$ and $\sigma_{1}=-1$. Thus, $\min \left\{j: j \geqq 1, \sigma_{j} \neq-1\right\}=k$ (as in $\S 3$ ). Thus, by $\S 4(\mathrm{~b})$, (assuming $\left.\alpha_{1}=\cdots=\alpha_{r}=\sigma_{0}\right)$,

$$
y_{1} \sim\left(\sigma_{k}+1\right)^{-1}\left(\log x \cdots \log _{k} x\right) Q^{*} .
$$

Since $\delta_{1}\left(y_{1}\right)=0$, we find by continuing up to $r$ using $\S 4$ (b) that $y_{r} \sim((r-1) !)^{-1}(\log x)^{r-1} y_{1}$. We now continue using $\S 4($ a) and find that $y_{p} \sim b_{p}\left(F^{(r)}\left(\sigma_{0}\right) / r !\right)^{-1} y_{r}$. Since $(r-1) !=s_{r, r-1}\left(\sigma_{1}+r\right)$, clearly $y_{p} \sim M$.

Case IV. $\sigma_{0}$ is a root of $F$ of multiplicity $r$ and $\sigma_{1} \in\{-2, \cdots,-r\}$. Let $s=-\sigma_{1}$. Since $\sigma_{1} \neq-1$, by $\S 4(\mathrm{~b})$, (assuming $\alpha_{1}=\cdots=\alpha_{r}=\sigma_{0}$ ), $y_{1} \sim\left(\sigma_{1}+1\right)^{-1}(\log x) Q^{*}$. Continuing up to $s-1$, we find

$$
y_{s-1} \sim\left[\left(\sigma_{1}+1\right) \cdots\left(\sigma_{1}+s-1\right)\right]^{-1}(\log x)^{s-1} Q^{*} .
$$

Since $\delta_{1}\left(y_{s-1}\right)=-1$, we have by $\S 4(\mathrm{~b})$,

$$
y_{s} \sim\left(\sigma_{k}+1\right)^{-1} \log x \cdots \log _{k} x y_{s-1} .
$$

Since $\delta_{1}\left(y_{s}\right)=0$, we have, using $\$ 4(\mathrm{~b})$, that $y_{r} \sim((r-s) !)^{-1}(\log x)^{r-s} y_{s}$. Now, using $\S 4(\mathrm{a})$, we find $y_{p} \sim b_{p}\left(F^{(r)}\left(\sigma_{0}\right) / r !\right)^{-1} y_{r}$. Since $(r-s)$ ! $=\left(\sigma_{1}+r\right) \cdots\left(\sigma_{1}+s+1\right)$, it follows that $y_{p} \sim M$.

6. Conclusion of main theorem (\$3). For each $i, B_{i}=b_{i}+w_{i}$ where $b_{i}=B_{i}(\infty)$ and $\delta_{0}\left(w_{i}\right)<0$. Letting $\Phi(y)=\sum_{i=0}^{n} b_{i} \theta^{i} y$ and $\Gamma(y)$ $=\sum_{i=0}^{n} w_{\imath} \theta^{i} y$, we have by $\S 5$ that there exists a function $y^{*} \sim M$ in $F(a, b)$ such that $\Phi\left(y^{*}\right)=\phi$. Under $y=y^{*}+z, \Omega(y)=\phi$ becomes $\Omega(z)=-\Gamma\left(y^{*}\right)$. Now if $\delta_{0}\left(y^{*}\right)=\lambda$, then it is easily verified that $\delta_{0}\left(\theta^{j} y^{*}\right) \leqq \lambda$ for each $j$. Letting $\epsilon>0$ be such that $\delta_{0}\left(w_{i}\right)<-\epsilon$ for each $i$, we have

$$
\delta_{0}\left(\Gamma\left(y^{*}\right)\right)<\lambda-\epsilon .
$$

We now utilize a technique employed by Strodt in [5] which we outline here for the reader's convenience. Let $H=\{\alpha: F(\alpha)=0\}$. Then if $q$ is a real number not in $H$ and we let $k_{q}=(F(q))^{-1}$, it is easily seen that the principal monomial of $\Omega(y)-x^{q}$ is $k_{q} x^{q}$. Hence if we let $\Lambda_{q}(\omega)=x^{-q} \Omega\left(k_{q} x^{q} \omega\right)$, then by the properties of a principal monomial we have $\Lambda_{q}(1) \sim 1$ and $\Lambda_{q}(E) \prec 1$ if $E \prec 1$. (Thus $\Lambda_{q}$ is unimajoral in the terminology of $\left[4\right.$, Section 13]). Further, it is easily seen that $\Lambda_{q}$ has coefficients in an $L D_{0}(F(a, b))$ and that $\partial \Lambda_{q} / \partial \omega^{(n)}$ is a nontrivial function. Thus by [4, Section 27], $\Lambda_{q}$ possesses at least one principal fac- 
torization sequence, that is, a sequence $\left(V_{1}, \cdots, V_{n}\right)$ of logarithmic monomials such that $\Lambda_{q}$ may be written

$$
\Lambda_{q}=\dot{V}_{n} \cdots \dot{V}_{1}+\sum_{j=0}^{n} E_{j} \dot{V}_{j} \cdots \dot{V}_{1}
$$

where $\dot{V}_{j}$ is the operator $\dot{V}_{j}(y)=y-y^{\prime} / V_{j}$ and where each $E_{j} \prec 1$. Now by definition of $\Lambda_{q}$, it is easily verified that

$$
\Lambda_{q}(\omega)=k_{q} \sum_{j=0}^{n} B_{j}(q+\theta)^{j} \omega,
$$

and so it follows from [4, Section 44] that all principal factorization sequences for $\Lambda_{q}(\omega)$ can be obtained as follows. If we let

$$
C_{1}^{*} \Lambda_{q}(y)=k_{q} \sum_{j=0}^{n} B_{j}(q+x y)^{j}
$$

and if $N_{1}, \cdots, N_{n}$ are the logarithmic monomials such that the zeros $y_{1}, \cdots, y_{n}$ of $C_{1}^{*} \Lambda_{q}(y)$ satisfy $y_{j} / N_{j} \rightarrow 1$ for each $j$, then $\left(V_{1}, \cdots, V_{n}\right)$ is a principal factorization sequence for $\Lambda_{q}$ if and only if $\left(V_{1}, \cdots, V_{n}\right)$ is a permutation of $\left(N_{1}, \cdots, N_{n}\right)$ and for each $j$, $V_{j}$ is either $\prec$ or $\approx$ to $V_{j+1}$. Since $\left\{B_{0}, \cdots, B_{n}\right\}$ is contained in an $L D_{0}(F(a, b))$, it easily follows that if $\left(V_{1}, \cdots, V_{n}\right)$ is a principal factorization sequence for $\Lambda_{q}$, then for each $j, V_{j}$ has the form

$$
V_{j}=c_{j} x^{-1+t_{j}}
$$

for some constant $c_{j}$ and some $t_{j} \geqq 0 . V_{j}$ is called nonexceptional if either $t_{j}>0$ or $c_{j}$ is not purely imaginary, and $\left(V_{1}, \cdots, V_{n}\right)$ is called nonexceptional if each $V_{j}$ is nonexceptional. From the definition of $C_{1}^{*} \Lambda_{q}(y)$, it follows that for $q$ and $s$ not in $H$, we have

$$
C_{1}^{*} \Lambda_{q}(y)=k_{q}\left(k_{s}\right)^{-1} C_{1}^{*} \Lambda_{s}\left((q-s) x^{-1}+y\right) .
$$

We now fix $s$ and we fix a principal factorization sequence for $\Lambda_{s}$ (which may be exceptional). By the above relation, $y^{*}$ is a zero of $C_{1}^{*} \Lambda_{q}(y)$ if and only if $(q-s) x^{-1}+y^{*}$ is a zero of $C_{1}^{*} \Lambda_{s}(y)$, and so it easily follows from the previous discussion that except for finitely many real $q, \Lambda_{q}(\omega)$ possesses a nonexceptional principal factorization sequence. Thus we have outlined the proof given in [5] that there is a finite set $G$ of real numbers such that for any real number $q \notin G$, $\Lambda_{q}(\omega)$ is unimajoral and possesses a nonexceptional principal factorization sequence.

In our case here, we choose a real number $q \notin G$ such that $\lambda-\epsilon$ $<q<\lambda$, and let $\left(V_{1}, \cdots, V_{n}\right)$ be a nonexceptional principal fac- 
torization sequence for this $\Lambda_{q}$. If $V_{j}$ has the form in (2), then its indicial function (as defined in [4, Section 61]) is the function defined on $(a, b)$ given by $f_{j}(\alpha)=\cos \left(t_{j} \alpha+\arg c_{j}\right)$. (Thus if $t_{j}=0, f_{j}$ is the constant function $\cos \left(\arg c_{j}\right)$.) Since each $V_{j}$ is nonexceptional, each $f_{j}$ has only finitely many zeros in $(a, b)$. Hence the union of the sets of roots of the $f_{j}$ is a finite set $\gamma_{1}<\cdots<\gamma_{d}$ in $(a, b)$. Thus if $I=\left(a_{1}, b_{1}\right)$ is any of the intervals $\left(a, \gamma_{1}\right),\left(\gamma_{1}, \gamma_{2}\right), \cdots,\left(\gamma_{d}, b\right)$, then no indicial function vanishes anywhere in $I$, so by definition $[4, \S 98],\left(V_{1}, \cdots\right.$, $\left.V_{n}\right)$ is unblocked in $\left(a_{1}, a_{2}, b_{1}\right)$ for any $a_{2}$. Furthermore, by (1) and choice of $q, x^{-q} \Gamma\left(y^{*}\right) \prec 1$, and so by definition [4, §88], $\left(V_{1}, \cdots, V_{n}\right)$ is a strong factorization sequence for $\Lambda_{q}(\omega)+x^{-q} \Gamma\left(y^{*}\right)$ in $F(I)$. Hence by $\left[4, \S 99\right.$, Theorem II], the equation $\Lambda_{q}(\omega)+x^{-q} \Gamma\left(y^{*}\right)=0$ possesses a solution $\omega_{0} \prec 1$ in $F(I)$. Letting $z_{0}=k_{q} x^{z} \omega_{0}$, we have $\Omega\left(z_{0}\right)=-\Gamma\left(y^{*}\right)$; and since $q<\lambda$, we have $z_{0} \prec y^{*}$. Letting $y_{0}=y^{*}+z_{0}$, we then have $\Omega\left(y_{0}\right)=\phi$ and $y_{0} \sim M$ in $F(I)$, which concludes the proof.

7. Corollary. Under the hypothesis and notation of $\$ 3$, let $I$ be a subinterval of $(a, b)$ such that in $F(I)$ there is a solution $y_{0} \sim M$ of $\Omega(y)=\phi$ (as just proved) and such that a complete logarithmic set of solutions $\left\{g_{1}, \cdots, g_{p}\right\}$ of $\Omega(y)=0$ exists in $F(I)$. (It was shown in $[1, \S 11]$ that if $p=\max \left\{j: B_{j}(\infty) \neq 0\right\}$, then e.f.d. in $F(a, b)$ there exist solutions $g_{1}, \cdots, g_{p}$ of $\Omega(y)=0$ such that $g_{j} \sim x^{\alpha}{ }_{j}(\log x)^{\beta_{j}}$ for some complex $\alpha_{j}$ and integer $\beta_{j}$ and such that $\left(\alpha_{k}, \beta_{k}\right) \neq\left(\alpha_{j}, \beta_{j}\right)$ if $k \neq j$.)

Then if $y^{*}$ is any solution of $\Omega(y)=\phi$ which in $F(I)$ is $\prec x^{\delta}$ for some constant $\delta$, then e.f.d. in $F(I)$ there exist constants $c_{1}, \cdots, c_{p}$ and $a$ trivial function $T(x)$ such that $y^{H}=y_{0}+\sum_{j=1}^{p} c_{i} g_{i}+T$.

Proof. $y^{*}-y_{0}$ is a solution of $\Omega(y)=0$ and is $\prec x^{a}$ for some $\alpha$ so the result follows immediately from $[1, \S 12]$.

\section{BIBLIOGRAPHY}

1. S. Bank, An asymptotic analog of the Fuchs regularity theorem, J. Math. Anal. Appl. 16 (1966), 138-151.

2. E. W. Chamberlain, Families of principal solutions of ordinary differential equations, Trans. Amer. Math. Soc. 107 (1963), 261-272.

3. W. Strodt, Contributions to the asymptotic theory of ordinary differential equations in the complex domain, Mem. Amer. Math. Soc. No. 13 (1954), 81 pp.

4. - Principal solutions of ordinary differential equations in the complex domain, Mem. Amer. Math. Soc. No. 26 (1957), 107 pp.

5. - Report on investigation in differential equations, Contract no. NSF G12984 between the NSF and Columbia University, November 1961.

6. - On the Briot and Bouquet theory of singular points of ordinary differential equations, Tech. Summary Rep. \#508, Math. Res. Ctr., U. S. Army, Univ. of Wis., 1964, $103 \mathrm{pp}$.

7. E. C. Titchmarsh, The theory of functions, Oxford Univ. Press, London, 1939.

UNIVERSITY of IlLiNoIS 